\title{
Delay-independent stability of $(Q, S, R)$-dissipative networked systems with a distributed controller
}

\author{
Tilemachos Matiakis and Sandra Hirche
}

\begin{abstract}
In this paper a distributed control approach aiming at delay-independent stability of networked $(\mathbf{Q}, \mathbf{S}, \mathbf{R})$ dissipative systems is proposed. The key idea is to exploit the available computational power in the plant side to locally implement a static-output-feedback-input-feedforward controller. This controller can be interpreted as an invertible transformation of arbitrary dissipativity properties of the plant into finite $L_{2}$-gain properties which are invariant against unknown constant time delay. As a result delay-independent stability of the overall system is guaranteed if the original plant and controller satisfy the well-known dissipativity-based stability conditions for interconnected systems without time delay. Extensions to time-varying delay and packet loss are straightforward. The superior performance of the proposed approach is demonstrated in simulations.
\end{abstract}

\section{INTRODUCTION}

In networked control systems (NCS) the spatially separated plant and controller are connected through a communication network, see Fig. 1. One of the numerous advantages of NCS compared to the standard architecture, is their flexible reconfiguration; nodes can be added or removed without additional wiring effort. However, the benefits of NCS come at the cost of time delay and possibly packet loss in the communication. The exact transmission characteristics typically depend on the number of nodes sharing the communication line as well as their transmission characteristics, and may be largely unknown during the controller design.

For a general overview in the NCS literature see [2]. Only a few approaches utilize the limited computational power which is available in the plant side. For instance in [11] the controller estimates and sends future values of its output together with the current one, and in the plant side a "time delay compensator" chooses the most recent value. An interesting approach appears also in [5] where signal processing techniques are used at plant and controller side to reduce the influence of communication unreliabilities modelled by a constant signal-to-noise ratio, however without time delay considerations.

In this paper the focus is on the stabilization in the presence of unknown, constant time delay. Constant time delay approaches are classified into delay-dependent and delay-independent, according to whether a bound on the time delay value is necessary or not for stability guarantees, for

This work was supported in part by the German Research Foundation (DFG) within the Priority Programme SPP 1305 "Regelungstheorie digital vernetzter dynamischer Systeme".

All authors are with the Institute of Automatic Control Engineering Technische Universität München D-80290 München, Germany www.lsr.ei.tum.de, T.Matiakis@tum.de, hircheetum.de

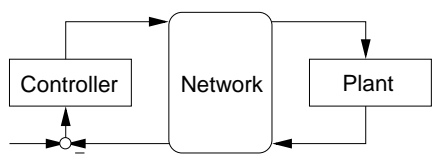

Fig. 1. Typical networked control system architecture.

a concise overview see [6]. A distributed control approach is proposed here for delay-independent stability, assuming that only a linear static-output-feedback-input-feedforward (SOFIF) controller can be implemented in the plant side. It should be noted that the proposed approach is straightforwardly extendable to time-varying delay and packet as explained later. We assume that there is a pre-designed controller for the system without taking into account the time delay. We additionally require that for zero time delay the response of the closed loop system is identical to that of system with the pre-designed controller, but without the communication network.

The plant and the pre-designed controller can be considered non-linear systems belonging to the class of $(Q, S, R)$ dissipative systems. Stability without the communication network is assumed to be guaranteed based on classical dissipativity theory results for interconnected dissipative systems [7]. The major contribution of this paper is a SOFIF controller for the plant side together with a modification of the original controller such that delay-independent stability is guaranteed. The SOFIF controller can be interpreted as an invertible transformation of the input-output behavior of the $(Q, S, R)$-dissipative plant. Roughly speaking, the SOFIF controller transforms the plant into a finite gain $L_{2}$ stable system. The constant time delay operator does not alter this $L_{2}$ gain. At the controller side another SOFIF system transforms the input-output behavior of the subsystem comprising the communication network and the plant with the SOFIF control such that it belongs to the same class of $(Q, S, R)$ dissipative systems as the plant. Stability is guaranteed based on the feedback interconnection of this subsystem and the pre-designed controller. An alternative interpretation uses small gain type arguments.

This work is originally inspired by the scattering transformation [1], [17], a method frequently encountered in force feedback telepresence systems with unknown, constant time delay, which bears however the restrictive assumption of passive plant and controller. This paper is a continuation of [14], [16] extending the approach in [16] to $(Q, S, R)$ dissipative systems.

The remainder of this paper is organized as follows. In Section II the necessary background on dissipativity is 
given. Section III presents the proposed distributed control architecture and in Section IV the main result regarding the delay-independent stability is provided. Simulations are performed in order to validate the proposed approach in Section V.

\section{BACKGROUND}

Notation. Let $\|u\|$ be the $L_{2}$ norm of a continuous time function $u(\cdot): \mathbb{R}_{+} \mapsto \mathbb{R}^{m}$, with $\mathbb{R}_{+}$being the set of real positive numbers and $\mathbb{R}^{m}$ the Euclidean space of dimension $m$. The truncation of $u(\cdot)$ until time $t$ is denoted by $u_{t}(\cdot)$. The inner product of the truncated signals $u_{t}, y_{t}$ is denoted by $\langle u, y\rangle_{t}$, hence $\left\|u_{t}\right\|^{2}=\langle u, u\rangle_{t}$. With $L_{2 e}^{m}$ the extended space of Lebesgue integrable functions of dimension $m$ with support on $\mathbb{R}_{+}$is denoted. We consider a dynamical system $\Sigma$ as a causal input-output mapping $\Sigma: \mathscr{U} \mapsto \mathscr{Y}$ where $\mathscr{U} \subset L_{2 e}^{m}, \mathscr{Y} \subset L_{2 e}^{p}$ the admissible input and output spaces respectively and $\Sigma(t \leq 0)=0$. The considered systems are well defined, i.e. to each element in $\mathscr{U}$ an element in $\mathscr{Y}$ is associated. The notation $M>0(" \geq ", "<"$, , $\leq$ ") denotes the positive definiteness (positive semi-definiteness, negative definiteness, negative semi-definiteness respectively) of $M$. With $\operatorname{diag}\left\{d_{i}\right\}$ a (block) diagonal matrix with elements $d_{i}$ is denoted, and with $I$ the unit matrix.

\section{A. Dissipativity}

For a detailed discussion on dissipativity see [7], [20]. Consider a system $\Sigma: \mathscr{U} \mapsto \mathscr{Y}$ where $\mathscr{U} \subset L_{2 e}^{m}, \mathscr{Y} \subset L_{2 e}^{p}$ and its input-output vector denoted by $z^{T}=\left[u^{T} y^{T}\right]$ where $u \in \mathscr{U}, y \in \mathscr{Y}$. Consider additionally the matrices $Q=Q^{T} \in$ $\mathbb{R}^{m \times m}, S \in \mathbb{R}^{p \times m}$ and $R=R^{T} \in \mathbb{R}^{p \times p}$.

Definition 1: A system $\Sigma: \mathscr{U} \mapsto \mathscr{Y}$ is called $(Q, S, R)$ dissipative if for each admissible $u \in \mathscr{U}$ and each $t \geq 0$ holds

$$
\begin{gathered}
\int_{0}^{t} z^{T} P z \mathrm{~d} \tau=\int_{0}^{t} u^{T} Q u+y^{T} R y+2 u^{T} S y \mathrm{~d} \tau \geq 0, \\
\text { where } P=\left[\begin{array}{cc}
Q & S \\
S^{T} & R
\end{array}\right] .
\end{gathered}
$$

Note, that we use the notation of [18] and denote the matrix related to the output by $R$ and the one related to the input by $Q$, contrary to [7], [20] where the inverse notation is used. Dissipativity defines a restriction on the input-output behavior of a system. The dissipativity matrix $P(2)$ is not unique for each system. For instance, if a system is $(Q, S, R)$ dissipative with a matrix $P$, it is also $(Q, S, R)$-dissipative with every matrix satisfying $P^{\prime} \geq P$. If the inequality holds strictly, $P^{\prime}$ is less restrictive, i.e. more conservative than $P$.

A special case of $(Q, S, R)$-dissipativity, with $S=0, R=-I, Q=\gamma^{2} I, \gamma \in \mathbb{R}_{+}$, is finite gain $L_{2}$ stability.

Definition 2: [8] A system $\Sigma: \mathscr{U} \mapsto \mathscr{Y}$ is called finite gain $L_{2}$ stable if there exists $\gamma \in \mathbb{R}_{+}$so that for each $u \in \mathscr{U}$ and each $t \geq 0$

$$
\left\|y_{t}\right\| \leq \gamma\left\|u_{t}\right\|
$$

Inequality (3) states that for each input, the output is bounded by the norm of the input signal scaled by a constant factor $\gamma$. It is straightforward to examine if a $(Q, S, R)$-dissipative system is finite gain $L_{2}$ stable.

Proposition 1: [7] A $(Q, S, R)$-dissipative system $\Sigma: \mathscr{U} \mapsto \mathscr{Y}$ is finite gain $L_{2}$ stable if $R<0$.
The most important fact of the dissipativity theory, is that it can guarantee the stability of interconnected systems based only on their dissipativity parameters.

Proposition 2: [7] The negative feedback interconnection of $\Sigma_{p}: u_{p}(\cdot) \mapsto y_{p}(\cdot)$ and $\Sigma_{c}: u_{c}(\cdot) \mapsto y_{c}(\cdot)$ with dissipativity parameters $Q_{p}, R_{p}, S_{p}$ and $Q_{c}, R_{c}, S_{c}$ respectively, is finite gain $L_{2}$ stable from the reference input $w$ to the output of the plant and controller $\left[\begin{array}{ll}y_{p}^{T} & y_{c}^{T}\end{array}\right]$ if

$$
\hat{R}=\left[\begin{array}{cc}
Q_{p} & S_{p} \\
S_{p}^{T} & R_{p}
\end{array}\right]+\left[\begin{array}{rr}
R_{c} & -S_{c}^{T} \\
-S_{c} & Q_{c}
\end{array}\right]<0
$$

In case of known LTI systems it is straightforward to examine if a system is $(Q, S, R)$-dissipative, using linear matrix inequalities (LMIs).

Proposition 3: [18] A controllable, LTI system, with state space matrices $A, B, C, D$ is $(Q, S, R)$-dissipative with the matrix $P(2)$ if and only if there exists $K=K^{T}>0$ so that

$$
\left[\begin{array}{rr}
A^{T} K+K A & K B \\
B^{T} K & 0
\end{array}\right]-\left[\begin{array}{rr}
0 & I \\
C & D
\end{array}\right]^{T} P\left[\begin{array}{cc}
0 & I \\
C & D
\end{array}\right] \leq 0 .
$$

\section{B. Spectral theorem}

A normal matrix is one for which $P^{T} P=P P^{T}$ holds. Symmetric matrices are always normal since $P=P^{T} \Rightarrow P^{T} P=P P^{T}=P^{2}$. According to the spectral theorem a matrix $P$ is normal if and only if it can be decomposed as $P=U^{T} \Lambda U$, where $U \in \mathbb{R}^{n \times n}$ an orthogonal matrix, i.e. $U U^{T}=I$, and $\Lambda=\operatorname{diag}\left\{\lambda_{1}, \lambda_{2}, \ldots\right\}$. The columns of $U$ are the eigenvectors of $P$. The entries of $\Lambda$ are the eigenvalues of $P$, which are in the same order as the eigenvectors are ordered in $U$. The order can be arbitrarily chosen.

\section{Distributed CONTROL ARCHITECTURE}

The plant $\Sigma_{p}: \mathscr{U}_{p} \mapsto \mathscr{Y}_{p}$ is a mapping from the input $u_{p} \in \mathscr{U}_{p} \subset L_{2 e}^{m}$ to the output $y_{p} \in \mathscr{Y}_{p} \subset L_{2 e}^{p}$. The controller comprises two parts. The local controller, placed at the plant side, is a linear SOFIF controller, see Fig. 2, described by $\left[\begin{array}{ll}u_{p}^{T} & v_{r}^{T}\end{array}\right]^{T}=D_{r}\left[\begin{array}{ll}u_{r}^{T} & y_{p}^{T}\end{array}\right]^{T}$, where $D_{r} \in \mathbb{R}^{(m+p) \times(m+p)}$ the static controller gain matrix. The remote controller $\Sigma_{l}:\left[\mathscr{W} \mathscr{V}_{l}\right] \mapsto \mathscr{U}_{l}$, is a mapping from the reference input $w \in \mathscr{W} \subset L_{2 e}^{p}$ and the left output of the communication network $v_{l} \in \mathscr{V}_{l} \subset L_{2 e}^{p}$, to the left input to the communication network $u_{l} \in \mathscr{U}_{l} \subset L_{2 e}^{m}$. The further structure of the remote controller seen in Fig. 2 is explained later.

The communication network is modelled as a forward and a backward time delay operator, $\mathscr{D}_{T_{1}}$ (controller to plant channel) and $\mathscr{D}_{T_{2}}$ (plant to controller channel) respectively, with time delays $T_{1}, T_{2} \in \mathbb{R}_{+}$constant but unknown. The input-output relations are given by $\mathscr{D}_{T_{1}}: u_{r}(t)=u_{l}\left(t-T_{1}\right)$ and $\mathscr{D}_{T_{2}}: v_{l}(t)=v_{r}\left(t-T_{2}\right)$. Their initial conditions are assumed to be zero:

$$
u_{r}(t)=0, t \in\left[-T_{1}, 0\right], \quad v_{l}(t)=0, t \in\left[-T_{2}, 0\right] .
$$

The pre-designed controller is denoted by $\Sigma_{c}: \mathscr{E} \mapsto \mathscr{Y}_{c}$ where $e \in \mathscr{E} \subset L_{2 e}^{p}$ the control error. As already mentioned, we require that for zero time delay the input-output behavior of the system is identical to that of the closed loop system with the pre-designed controller, and without the communication network. This, together with the fact that the controller $D_{r}$ 
is applied in the plant side, leads to the decomposition of $\Sigma_{l}$ depicted in Fig. 2.

We represent the local static controller $D_{r}$ as an invertible mapping between the right transmitted values $z_{r}^{T}=\left[\begin{array}{ll}u_{r}^{T} & v_{r}^{T}\end{array}\right]$ and the input-output plant vector $z_{p}^{T}=\left[\begin{array}{ll}u_{p}^{T} & y_{p}^{T}\end{array}\right]$, i.e. $z_{r}=M z_{p}$ with $\operatorname{det} M \neq 0$. The relation between $D_{r}$ and $M$ is given later. Consider now the system without the network, i.e. the feedback interconnection of $\Sigma_{l}$ and the subsystem $\Sigma_{r}: \mathscr{U}_{r} \mapsto \mathscr{V}_{r}$ where $u_{r} \in \mathscr{U}_{r}, v_{r} \in \mathscr{V}_{r}$.

Proposition 4: The feedback interconnection of the subsystems $\Sigma_{r}$ and $\Sigma_{l}$ is equivalent to the negative feedback interconnection of the systems $\Sigma_{p}$ and $\Sigma_{c}$, if $\Sigma_{l}$ can be decomposed into a static controller $D_{l}$, i.e. $\left[\begin{array}{ll}u_{l}^{T} & u_{c}^{T}\end{array}\right]^{T}=D_{l}\left[\begin{array}{ll}y_{c}^{T} & v_{l}^{T}\end{array}\right]^{T}$, $D_{l} \in \mathbb{R}^{(m+p) \times(m+p)}$, which represents the inverse mapping $M^{-1}$ between the input-output vectors $z_{c}^{T}=\left[\begin{array}{ll}y_{c}^{T} & u_{c}^{T}\end{array}\right]$, $z_{l}^{T}=\left[\begin{array}{ll}u_{l}^{T} & v_{l}^{T}\end{array}\right]$, i.e. $z_{c}=M^{-1} z_{l}$ and the system $\Sigma_{c}: \mathscr{E} \mapsto \mathscr{Y}_{c}$, where $e=w-u_{c}$, see Fig. 2 .

Proof: Considering that for zero time delay, i.e. when $z_{l}=z_{r}$ holds, the left static system cancels the right, i.e. $z_{c}=M^{-1} z_{l}=M^{-1} z_{r}=M^{-1} M z_{p}=z_{p}$.

The relations between $D_{r}, D_{l}$ and $M$ are

$$
\begin{aligned}
& D_{r}=\left[\begin{array}{ll}
D_{r 11} & D_{r 12} \\
D_{r 21} & D_{r 22}
\end{array}\right]=\left[\begin{array}{rr}
M_{11}^{-1} & -M_{11}^{-1} M_{12} \\
M_{21} M_{11}^{-1} & M_{22}-M_{21} M_{11}^{-1} M_{12}
\end{array}\right] \\
& D_{l}=\left[\begin{array}{ll}
D_{l 11} & D_{l 12} \\
D_{l 21} & D_{l 22}
\end{array}\right]=\left[\begin{array}{rr}
M_{11}-M_{12} M_{22}^{-1} M_{21} & M_{12} M_{22}^{-1} \\
-M_{22}^{-1} M_{21} & M_{22}^{-1}
\end{array}\right],
\end{aligned}
$$

where $\quad M_{11}, D_{r 11}, D_{l 11} \in \mathbb{R}^{m \times m}, \quad M_{12}, D_{r 12}, D_{l 12} \in \mathbb{R}^{m \times p}$, $M_{21}, D_{r 21}, D_{l 21} \in \mathbb{R}^{p \times m}, \quad M_{22}, D_{r 22}, D_{l 22} \in \mathbb{R}^{p \times p}$. Note that (6) require that $M_{11}=D_{r 11}$ is invertible. Additionally, the invertibility of $M$ implies that $M_{22}-M_{21} M_{11}^{-1} M_{12}=D_{r 22}$ is invertible. The invertibility of the $D_{r 11}, D_{r 22}$ guarantees that the controllability and observability of $\Sigma_{r}$ is preserved.

Proposition 5: Assume $\Sigma_{p}$ to be controllable and observable. The subsystem $\Sigma_{r}$ is guaranteed to be controllable if and only if $D_{r 11}$ is invertible. The system $\Sigma_{r}$ is guaranteed to be observable if and only if $D_{r 22}$ is invertible.

Proof: For the relation between the inputs of $\Sigma_{r}$ and $\Sigma_{p}$ we have $u_{p}=D_{r 11} u_{r}+D_{r 12} y_{p} \Rightarrow u_{r}=D_{r 11}^{-1}\left(u_{p}-D_{r 12} y_{p}\right)$, i.e. for each input $u_{p}$ and output $y_{p}$ we can find a signal $u_{r}$. Hence, if the system is controllable from the input $u_{p}$ it is also controllable from the input $u_{r}$. That controllability of $\Sigma_{r}$ may be lost if $D_{r 11}$ is not invertible is straightforward, if we consider $D_{r 11}=0$, meaning that no control signal reaches the plant input. The proof for observability follows the same line, considering $D_{r 22}$.

Example 1: The elements of $M$ in case of the scattering transformation and SISO systems are $M_{11}=\sqrt{b / 2}, M_{12}=$ $\sqrt{1 / 2 b}, M_{21}=-\sqrt{b / 2}, M_{22}=\sqrt{1 / 2 b}$, where $b>0$ a parameter that can be freely chosen [17]. Considering for simplicity $b=1$ the static controller gains (6) become

$$
D_{r}=\frac{1}{\sqrt{2}}\left[\begin{array}{rr}
1 & 1 \\
-1 & 2
\end{array}\right], \quad D_{l}=\frac{1}{\sqrt{2}}\left[\begin{array}{ll}
2 & 1 \\
1 & 1
\end{array}\right] .
$$

Note that for $M=I$ the standard approach without controller at the plant side is recovered. The following analysis deals with the design of $D_{r}, D_{l}$, or equivalently the selection of $M$, so that stability with unknown constant time delay is guaranteed. Throughout the paper we assume that the

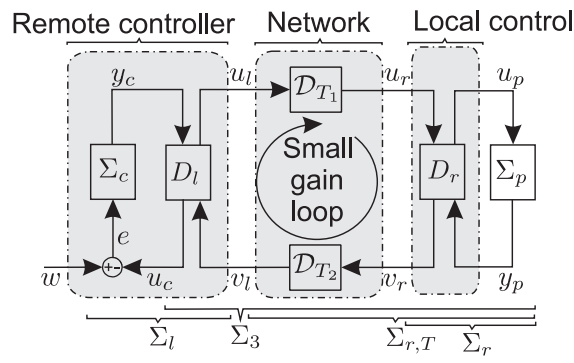

Fig. 2. Networked control system with local and remote control measures.

closed loop system is well posed, i.e. for each input $w \in \mathscr{W}$ there exists a unique solution for the signals $e, u_{c}, y_{c}, u_{l}, v_{l}$, $u_{r}, v_{r}, u_{p}, y_{p}$ that causally depend on $w$.

We make furthermore the following assumption:

A1. The plant $\Sigma_{p}$ and the controller $\Sigma_{c}$ are $(Q, S, R)$ dissipative systems with the matrices

$$
P_{p}=\left[\begin{array}{ll}
Q_{p} & S_{p} \\
S_{p}^{T} & R_{p}
\end{array}\right], \quad P_{c}=\left[\begin{array}{ll}
Q_{c} & S_{c} \\
S_{c}^{T} & R_{c}
\end{array}\right]
$$

respectively, satisfying Proposition 2 .

For further reference we define also the subsystems $\Sigma_{r, T}: \mathscr{U}_{l} \mapsto \mathscr{V}_{l}$ with $u_{l} \in \mathscr{U}_{l}, v_{l} \in \mathscr{V}_{l}$, which consists of $\Sigma_{r}$ and the communication network, and $\Sigma_{3}: \mathscr{Y}_{c} \mapsto \mathscr{U}_{c}$ with $y_{c} \in \mathscr{Y}_{c}, u_{c} \in \mathscr{U}_{c}$ comprising the plant, $D_{r}, D_{l}$ and the communication network, see Fig. 2.

\section{MAin RESUlt}

The description of the static systems $D_{r}, D_{l}$ by the matrix $M$ plays a significant role in the following. $M$ describes an invertible transformation of the $(Q, S, R)$-dissipativity properties of a system as stated in the following Lemma. Consider the plant $\Sigma_{p}$ and the subsystem $\Sigma_{r}$, see Fig. 2.

Lemma 1: The system $\Sigma_{r}$ is $(Q, S, R)$-dissipative with the matrix $P_{r}=M^{-T} P_{p} M^{-1}$ if and only if $\Sigma_{p}$ is $(Q, S, R)$ dissipative with the matrix $P_{p}$.

Proof: The relation between the plant input-output vector $z_{p}$ and the input-output vector of $\Sigma_{r} z_{r}^{T}=\left[\begin{array}{ll}u_{r}^{T} & v_{r}^{T}\end{array}\right]$ is

$$
z_{p}=M^{-1} z_{r} \text {. }
$$

By substituting (7) in the plant dissipativity inequality (1) we get equivalently $\int_{0}^{t} z_{r}^{T} M^{-T} P_{p} M^{-1} z_{r} \mathrm{~d} \tau \geq 0$, which is exactly (1) for the input-output vector $z_{r}$ of $\Sigma_{r}$, and the dissipativity matrix $P_{r}=M^{-T} P_{p} M^{-1}$. Going the other way around, starting from $\Sigma_{r}$ with the dissipativity matrix $P_{r}$, considering that $z_{r}=M z_{p}$, and using the same procedure, we reach for $\Sigma_{p}$ the dissipativity matrix $P_{p}$.

A transformation of the form $N=M^{T} B M$ where $B, M$ are square matrices and $\operatorname{det} M \neq 0$, is called a congruence transformation. According to Sylvester's law of inertia congruence transformations do not change the inertia of a matrix, i.e the number of positive, negative and zero eigenvalues. In addition, between two matrices with the same inertia, a congruence transformation always exists. Thus, linear SOFIF control is sufficient to arbitrarily change the $(Q, S, R)$ dissipativity parameters of a system, as long as the inertia of the initial and the final dissipativity matrices are the same. 


\section{A. Delay-independent stability}

For the following analysis we decompose the closed loop system into the feedback interconnection of the systems $\Sigma_{c}$ and $\Sigma_{3}$, see Fig. 2. The underlying idea is that if the dissipativity property of the plant $\Sigma_{p}$ is preserved to $\Sigma_{3}$, then stability of the overall system can be guaranteed based on Assumption A1. We denote by $\lambda_{i}, \lambda_{i} \geq \lambda_{i+1}$ the eigenvalues of the plant dissipativity matrix $P_{p}$ and by $L$ the number of its non-negative eigenvalues. We denote further by $U_{p}$ a matrix having as columns the eigenvectors of $P_{p}$, ordered so that the corresponding eigenvalues are in decreasing order. By $D=\operatorname{diag}\left\{d_{i}\right\}>0$ an arbitrary diagonal matrix is denoted. The next Lemma presents the main result of this paper.

Lemma 2: If the dissipativity matrix of the plant $P_{p}$ has equal or less non-negative eigenvalues than the size of its input, i.e. $L \leq m$, and $M$ is chosen to be $M=D U_{p}$, then $\Sigma_{3}: \mathscr{Y}_{c} \mapsto \mathscr{U}_{c}$ is $(Q, S, R)$-dissipative with the matrix

$$
\begin{gathered}
P_{3}=P_{p}+U_{p}^{T} \Delta U_{p}, \\
\text { with } \Delta=\left[\begin{array}{cc}
\operatorname{diag}\left\{0, \ldots, 0,-\lambda_{L+1}, \ldots,-\lambda_{m}\right\} & 0 \\
0 & 0
\end{array}\right] \geq 0 .
\end{gathered}
$$

Proof: Based on the spectral theorem, the dissipativity matrix of the plant can be decomposed as $P_{p}=U_{p}^{T} \Lambda_{p} U_{p}$, with $\Lambda_{p}=\operatorname{diag}\left\{\lambda_{1}, \ldots, \lambda_{m+p}\right\}$. According to Lemma 1 , for $M=D U_{p}$ the dissipativity matrix of $\Sigma_{r}$ becomes

$$
P_{r}=D^{-T} U_{p}^{-T} U_{p}^{T} \Lambda_{p} U_{p} U_{p}^{-1} D^{-1}=D^{-T} \Lambda_{p} D^{-1} .
$$

Since $\Delta \geq 0(9), \Sigma_{r}$ is also $(Q, S, R)$-dissipative with

$$
P_{\text {diag }, r}=D^{-T} \Lambda_{p} D^{-1}+D^{-T} \Delta D^{-1} \text {. }
$$

The dissipativity inequality for $P_{\text {diag, } r}$ can be written as

$$
\begin{aligned}
& d_{m+1}^{2}\left(-\lambda_{m+1}\right)\left\|v_{r, t}^{1}\right\|^{2}+\ldots+d_{m+p}^{2}\left(-\lambda_{m+p}\right)\left\|v_{r, t}^{p}\right\|^{2} \leq \\
& d_{1}^{2} \lambda_{1}\left\|u_{r, t}^{1}\right\|^{2}+\ldots+d_{L}^{2} \lambda_{L}\left\|u_{r, t}^{L}\right\|^{2}
\end{aligned}
$$

where $u_{r}^{i}, v_{r}^{j}$ the $i$ input and $j$ output of the subsystem $\Sigma_{r}$ respectively, and $\left\{\lambda_{1}, \ldots, \lambda_{L}\right\}$ the non-negative eigenvalues. For the forward and backward constant time delay operators, taking into account (5) we can state

$$
\left\|u_{r, t}^{i}\right\|^{2} \leq\left\|u_{l, t}^{i}\right\|^{2},\left\|v_{l, t}^{j}\right\|^{2} \leq\left\|v_{r, t}^{j}\right\|^{2}, \quad \forall t>0,
$$

and substituting (13) in (12) it follows for the subsystem $\Sigma_{r, T}$

$$
\begin{aligned}
& d_{m+1}^{2}\left(-\lambda_{m+1}\right)\left\|v_{l, t}^{1}\right\|^{2}+\ldots+d_{m+p}^{2}\left(-\lambda_{m+p}\right)\left\|v_{l, t}^{p}\right\|^{2} \leq \\
& d_{1}^{2} \lambda_{1}\left\|u_{l, t}^{1}\right\|^{2}+\ldots+d_{L}^{2} \lambda_{L}\left\|u_{l, t}^{L}\right\|^{2},
\end{aligned}
$$

i.e. the dissipativity matrix of $\Sigma_{r, T}$ is $P_{r, T}=P_{\text {diag, } r}$. Applying Lemma 1 to $P_{r, T}=P_{\text {diag }, r}$ the Lemma is proved.

The system $D_{r}$ transforms the plant $\Sigma_{p}$ into the $(Q, S, R)$ dissipative system $\Sigma_{r}$ with a diagonal dissipativity matrix $P_{r}(10)$. If $P_{p}$, and consequently $P_{r}$, has less non-negative eigenvalues than the size of its input, then all the elements in the lower block diagonal part of $P_{r}$ which corresponds to the output are negative. Accordingly, there is a diagonal matrix, with negative elements in the lower diagonal part, and non-negative in the upper, i.e. $P_{\text {diag, } r}(11)$, for which the system is again $(Q, S, R)$-dissipative since $P_{\text {diag }, r} \geq P_{r}$ holds. The constant time delay operators do not affect this property, i.e. $\Sigma_{r, T}$ is also $(Q, S, R)$-dissipative with $P_{\text {diag, } r}$. The left static system $D_{l}$ transforms $\Sigma_{r, T}$ into another $(Q, S, R)$-dissipative system with its dissipativity matrix $P_{3}$ depending directly on the dissipativity matrix of the plant $P_{p}$.
Since $\Delta \geq 0 P_{3}$ is in general more conservative than $P_{p}$. The matrix $\Delta$ becomes zero if the matrix $P_{p}$ has exactly $p$ negative eigenvalues. In this case, $P_{3}=P_{p}$ holds, i.e. the exact same dissipativity property of the plant is inherited to $\Sigma_{3}$. In the remainder of this paper it is assumed that $M=D U_{p}$. For this selection of $M$ it is straightforward to formulate a stability condition.

Theorem 1: The closed loop system with constant time delay is finite gain $L_{2}$ stable from the reference input $w$ to the output of the plant $y_{p}$ if

$$
\hat{R}+U_{p}^{T} \Delta U_{p}<0
$$

where $\hat{R}$ is given by (4) based on Assumption A1.

Proof: For the feedback interconnection $\Sigma_{c}$ and $\Sigma_{3}$, based on Proposition 2 and substituting (8) (4) it follows

$$
\hat{R}_{3}=P_{3}+\left[\begin{array}{rr}
R_{c} & -S_{c}^{T} \\
-S_{c} & Q_{c}
\end{array}\right]=\hat{R}+U_{p}^{T} \Delta U_{p} .
$$

In consequence, $\hat{R}+U_{p}^{T} \Delta U_{p}<0 \Rightarrow \hat{R}_{3}<0$, i.e. the closed loop system is finite gain $L_{2}$ stable from the input $w$ to $z_{c}^{T}=\left[\begin{array}{ll}y_{c}^{T} & u_{c}^{T}\end{array}\right]$, i.e. the signals $u_{c}, y_{c}, e \in L_{2 e}$. Since $u_{l}, v_{l}$ are linear combinations of $u_{c}, y_{c}$ we have $u_{c}, y_{c} \in L_{2 e} \Rightarrow$ $u_{l}, v_{l} \in L_{2 e}$. The forward time delay operator is finite gain $L_{2}$ stable so $u_{l} \in L_{2 e} \Rightarrow u_{r} \in L_{2 e}$. Further the subsystem $\Sigma_{r}$ is finite gain $L_{2}$ stable, thus $u_{r} \in L_{2 e} \Rightarrow v_{r} \in L_{2 e}$. Since $u_{p}, y_{p}$ are a linear transformation of $u_{r}, v_{r}$, we have that $u_{r}, v_{r} \in L_{2 e} \Rightarrow u_{p}, y_{p} \in L_{2 e}$, i.e. there exists a $\gamma<\infty$ such that $\left\|y_{p, t}\right\| \leq \gamma\left\|w_{t}\right\|$ holds $\forall t$.

Stability of the overall system is guaranteed if $\hat{R}$ is "enough negative definite" so that $\hat{R}<-U_{p}^{T} \Delta U_{p}$ holds. In case the plant dissipativity matrix has $p$ negative eigenvalues $\hat{R}_{3}=\hat{R}$ holds. In consequence, every plant controller pair which is stable without the network, is again stable with the network with arbitrarily large constant time delay and the proposed transformation. Note, that the elements of $D$ can be freely chosen to meet performance requirements.

\section{B. Small gain interpretation}

For a specific choice of the elements of the matrix $D$ the interpretation of Theorem 1 from a small gain perspective, gives an interesting viewpoint of the proposed approach. Therefore the closed loop system is decomposed into the subsystems $\Sigma_{r}, \Sigma_{l}, \mathscr{D}_{T_{1}}$, and $\mathscr{D}_{T_{2}}$ where the transmitted signals $u_{l}, u_{r}, v_{r}, v_{l}$ act as inputs and outputs. The open loop system $\Sigma_{O L}=\Sigma_{l} \circ \mathscr{D}_{T_{1}} \circ \Sigma_{r} \circ \mathscr{D}_{T_{2}}$, is considered, see Fig. 2, with its $L_{2}$ gain denoted by $\gamma_{O L}$.

Corollary 1: If the elements of $D$ are chosen by

$$
d_{i}=\left\{\begin{array}{l}
\sqrt{\frac{q_{r}}{\left|\lambda_{i}\right|}}, i \in\{1, \ldots, m\}, \\
\sqrt{\frac{r_{r}}{\left|\lambda_{i}\right|}}, i \in\{m+1, \ldots, m+p\}
\end{array},\right.
$$

with $q_{r}, r_{r}>0$, the system $\Sigma_{O L}$ has an $L_{2}$ gain $\gamma_{O L}<1$.

Proof: For the constant time delay operators we have $\gamma_{T_{1}}, \gamma_{T_{2}}=1$, thus, for the $L_{2}$ gain of $\Sigma_{O L}$ holds $\gamma_{O L} \leq \gamma_{l} \gamma_{r} \gamma_{T_{1}} \gamma_{T_{2}}=\gamma_{l} \gamma_{r}$. It remains to show that $\gamma_{l} \gamma_{r}<1$. Choosing $d_{i}$ from (15) the dissipativity matrix of $\Sigma_{r}$ (11) becomes $P_{\text {diag }, r}=\operatorname{diag}\left\{q_{r} I,-r_{r} I\right\}$, i.e. $\Sigma_{r}$ is finite gain $L_{2}$ stable with gain $\gamma_{r}^{2}=\frac{q_{r}}{r_{r}}$. Considering that $u_{c}=-e$ the dissipativity inequality (1) for $\Sigma_{c}$ with the input-output vector $z_{c}^{T}=\left[\begin{array}{ll}y_{c}^{T} & -e^{T}\end{array}\right]$ becomes 


$$
\int_{0}^{t} z_{c}^{T} P_{c}^{\prime} z_{c} \mathrm{~d} \tau \geq 0 \text { with } P_{c}^{\prime}=\left[\begin{array}{rr}
R_{c} & -S_{c}^{T} \\
-S_{c} & Q_{c}
\end{array}\right] .
$$

Considering that $z_{c}=M^{-1} z_{l}$ and according to Lemma 1 for the dissipativity matrix of the subsystem $\Sigma_{l}$ we have $P_{l}=M^{-T} P_{c}^{\prime} M^{-1}$, and substituting $P_{c}^{\prime}=-P_{p}+\hat{R}$ from (4) and (11), it follows $P_{l}=-P_{\text {diag, } r}+M^{-T}\left(\hat{R}+U_{p}^{T} \Delta U_{p}\right) M^{-1}$. Subsituting (14) we have $P_{l}<-P_{\text {diag }, r}=\operatorname{diag}\left\{-q_{r} I, r_{r} I\right\}$, i.e. for the $L_{2}$ gain of $\Sigma_{l}$ it holds $\gamma_{l}^{2}<\frac{r_{r}}{q_{r}}=\frac{1}{\gamma_{r}^{2}} \Leftrightarrow \gamma_{l}^{2} \gamma_{r}^{2}<1$.

Hence, the small gain condition in the communicated variables is satisfied. In fact, with equality in Theorem 1 i.e. marginal stability, also the open loop gain becomes $\gamma_{O L}=1$. If (4) is more robustly satisfied it results in smaller gain $\gamma_{r} \gamma_{l}$, i.e. a larger stability reserve. Note, that the small gain theorem does not need to be satisfied between the mappings with the (original) control variables $e, y_{c}, u_{p}, y_{p}$.

Remark 1: In case of unstable plants the proposed approach locally pre-stabilizes the plant, by the righthand static controller $D_{r}$. This becomes clear from the fact that for the dissipativity matrix of $\Sigma_{r}, R_{r}<0$ holds, where $R_{r}$ its lower block diagonal part, i.e. $\Sigma_{r}$ is finite gain $L_{2}$ stable.

Remark 2: Observe that the only important fact for the stability is that for the $L_{2}$ gain of the time delay operators $\gamma_{T_{1}}, \gamma_{T_{2}} \leq 1$ holds. Hence, replacing the time delay operators $\mathscr{D}_{T_{1}}, \mathscr{D}_{T_{2}}$ with any other norm bounded uncertainty with $\gamma \leq 1$ does alter the stability result. Many approaches for time-varying delay [13] and packet loss [3] in case of the scattering transformation use this fact, introducing control actions to keep the $L_{2}$ gain of the network input-output operator to $\gamma \leq 1$. These approaches are straightforward to apply here.

Remark 3: For input-feedforward-output-feedbackpassive systems, i.e. systems with $Q=-\delta I, R=-\varepsilon I$, $S=\frac{1}{2} I, \delta, \varepsilon \in \mathbb{R}$, a matrix $M$ which exactly preserves the dissipativity properties of the plant always exists, as $L=m$ holds. This matrix can be analytically computed, see [16].

Remark 4: In case of passive systems, i.e. $Q=R=0, S=\frac{1}{2} I$, the spectral theorem gives

$$
U_{p}=\frac{\sqrt{2}}{2}\left[\begin{array}{rr}
I & I \\
-I & I
\end{array}\right]
$$

By choosing further $D=\operatorname{diag}\left\{\sqrt{b} I,(\sqrt{b})^{-1} I\right\}$, the well known scattering transformation [1], [17] is recovered.

\section{Dissipativity parameters for known LTI system}

If plant and controller are LTI systems with state space matrices $A_{i}, B_{i}, C_{i}, D_{i}, i \in\{p, c\}$, LMIs can be used to examine if there are matrices $P_{p}, P_{c}$ satisfying Assumption A1.

Proposition 6: The plant $\Sigma_{p}$ and the controller $\Sigma_{c}$ are $(Q, S, R)$-dissipative with $P_{p}, P_{c}$, satisfying Proposition 2 , if and only if there are $K_{i}=K_{i}^{T}>0, i \in\{p, c\}$ so that

$$
\begin{gathered}
{\left[\begin{array}{rr}
A_{i}^{T} K_{i}+K_{i} A_{i} & K_{i} B_{i} \\
B_{i}^{T} K_{i} & 0
\end{array}\right]-\left[\begin{array}{rr}
0 & I \\
C_{i} & D_{i}
\end{array}\right]^{T} P_{i}\left[\begin{array}{rr}
0 & I \\
C_{i} & D_{i}
\end{array}\right] \leq 0,} \\
{\left[\begin{array}{rr}
Q_{p} & S_{p} \\
S_{p}^{T} & R_{p}
\end{array}\right]+\left[\begin{array}{rr}
R_{c} & -S_{c}^{T} \\
-S_{c} & Q_{c}
\end{array}\right]<0 .}
\end{gathered}
$$

Proof: The proof is straightforward by combining Propositions 3 and 2.

Remark 5: Proposition 6 leads to only a sufficient delayindependent stability condition, as it is based on the sufficient condition of Proposition 2. The necessary and sufficient delay-independent stability condition is based on a small gain condition of $\Sigma_{r} \circ \Sigma_{l}$, i.e. $\gamma_{\Sigma_{r}} \circ \Sigma_{l}<1$. The conservatism is reduced because $\gamma_{\Sigma_{r} \circ \Sigma_{l}} \leq \gamma_{r} \gamma_{l}$ holds in general with strict inequality. However, the direct design of $D_{r}, D_{l}$ so that $\gamma_{\Sigma_{r} \circ \Sigma_{l}}<1$ holds is complicated, because $D_{r}, D_{l}$ are coupled through the matrix $M$ (6). In [15] this has been formulated as a problem with bilinear matrix inequalities but only for the single-input-single-output case.

\section{NumeriCAL EXAMPLE}

We consider the PSM example, extracted from the publicly available benchmark collection $\mathrm{COMPl}_{e}$ ib [10] described by

$$
\begin{aligned}
& \dot{x}_{p}=A_{p} x_{p}+B_{1} w+B_{p} u_{p}, \\
& z_{p}=C_{1} x_{p}+D_{11} w_{p}+D_{12} u_{p}, \quad y_{p}=C_{p} x_{p}+D_{12} w_{p}
\end{aligned}
$$

where $x_{p}(\cdot) \in \mathbb{R}^{7}$ the states, $u_{p} \in \mathbb{R}^{2}, y_{p} \in \mathbb{R}^{3}$ the control input and output, and $w_{p} \in \mathbb{R}^{2}, z_{p} \in \mathbb{R}^{5}$ the external input and output respectively. Three different controllers are compared, a linear quadratic regulator (LQR) with and without the static systems $D_{r}, D_{l}$, and a state feedback controller satisfying the small gain condition. It should be pointed out, that the comparison to the small gain based controller is "unfair", since state feedback is required contrary to the other two controllers which consider output feedback.

\section{A. Controller design}

As the controller $\Sigma_{c}$ a LQR for zero time delay is considered minimizing the cost function

$$
J=\left\|z_{p}\right\|^{2}+0.1\left\|u_{p}\right\|^{2} \mathrm{~d} \tau
$$

together with a full state observer, with its poles placed in the positions $[-10,-11, \ldots,-16]$, so as to be ten times faster than the slowest pole of $\Sigma_{p}$. For the selection of $M$ Proposition 6 is solved with YALMIP Matlab toolbox [12] and the SDPT3 solver [19], in order to compute the plant dissipativity matrix $P_{p}$. The transformation $M$ is chosen according to Lemma 2 to be $M=U_{p}$, i.e. $D=I$ :

$$
M=\left[\begin{array}{ccccc}
-795 & -201 & 546 & 51 & 162 \\
-179 & 763 & 181 & 120 & -580 \\
235 & -492 & 389 & -325 & -667 \\
0.457 & 313 & 673 & -224 & 433 \\
266 & -189 & 251 & 909 & -63
\end{array}\right] * 10^{-3} .
$$

For the small gain based controller the LQR state feedback problem is formulated in LMIs [4], with an additional small gain constraint of the open loop transfer function, which ensures delay-independent stability:

\section{minimize $x_{0} \mathbf{K}_{\mathbf{1}} x_{0}$ subject to}

$$
\begin{aligned}
& {\left[\begin{array}{ll}
0.1 I & B_{p}^{T} \mathbf{K}_{\mathbf{1}} \\
\mathbf{K}_{\mathbf{1}} B_{p} & A_{p}^{T} \mathbf{K}_{\mathbf{1}}+\mathbf{P}_{\mathbf{1}} A_{p}+C_{p}^{T} C_{p}
\end{array}\right]<0, \mathbf{K}_{\mathbf{1}}>0, \mathbf{K}_{\mathbf{2}}>0, } \\
& {\left[\begin{array}{ll}
A_{p}^{T} \mathbf{K}_{\mathbf{2}}+\mathbf{K}_{\mathbf{2}} A_{p}+\mathbf{K}_{\mathbf{1}} B_{p} R^{-2} B_{p}^{T} \mathbf{K}_{\mathbf{1}} & \mathbf{K}_{\mathbf{2}} B_{p} \\
B_{p}^{T} \mathbf{K}_{\mathbf{2}} & -I
\end{array}\right]>0, } \\
\text { where } & \text { with bold letters the optimization parameters are }
\end{aligned}
$$
denoted, and $x_{p}(t=0)=\left[\begin{array}{lll}1 & \ldots & 1\end{array}\right]$ is the initial condition. Using the YALMIP Matlab toolbox [12] and the local solver PENBMI [9] the (locally optimal) state feedback is

$$
F=\left[\begin{array}{ccccccc}
289 & 72 & 299 & -227 & 206 & 61 & 251 \\
206 & 61 & 249 & 522 & 239 & 52 & 219
\end{array}\right] * 10^{-3} .
$$



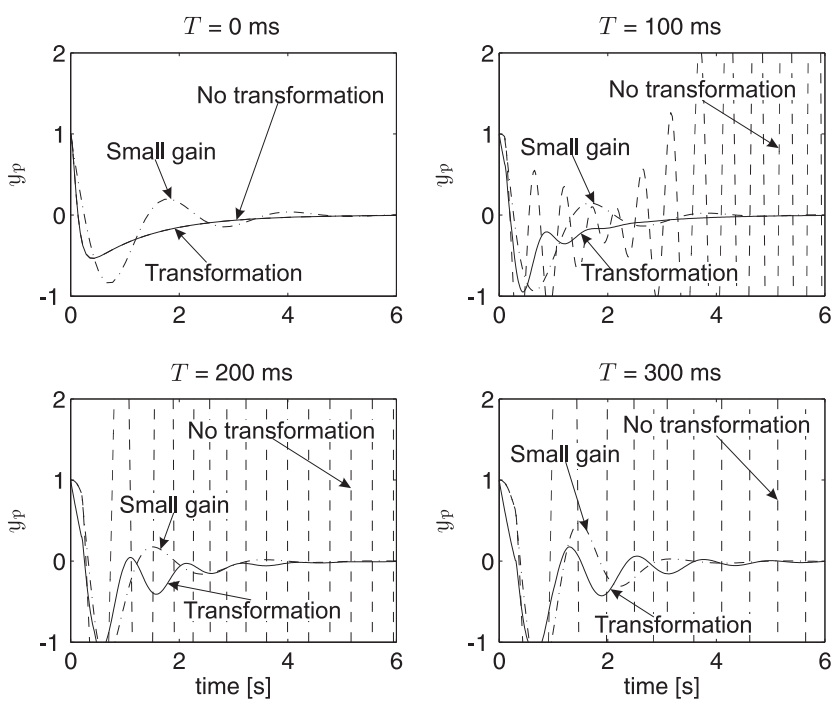

Fig. 3. Response of the first output of the systems with the LQR with and without the static controllers, and the small gain based controller, starting from the initial condition $x_{p}(t=0)=\left[\begin{array}{lll}1 & \ldots & 1\end{array}\right]^{T}$, for various time delay values.

$$
\text { TABLE I }
$$

OPTIMIZATION CRITERION FOR TIME HORIZON 6SEC.

\begin{tabular}{|c||c||c||c||c|}
\hline Time Delay $T_{1}+T_{2}[\mathbf{m s}]$ & $\mathbf{0}$ & $\mathbf{1 5 0}$ & $\mathbf{3 0 0}$ & $\mathbf{4 5 0}$ \\
\hline Transformation & 1.7377 & 1.9788 & 2.2330 & 2.4241 \\
\hline LQR & 1.7376 & unst. & unst. & unst. \\
\hline State feedback small gain & 1.7768 & 1.9584 & 2.3975 & 3.1788 \\
\hline
\end{tabular}

\section{B. Simulations}

For comparison the response of the systems is presented starting from the initial condition $x_{p}(t=0)=\left[\begin{array}{lll}1 & \ldots & 1\end{array}\right]^{T}$, see Fig.3. Due to space limitations only the first output is depicted. Various values for the round trip time delay $T=$ $T_{1}+T_{2}$ are considered, equally divided to the forward and the backward path. The system with the proposed approach remains stable and its response is only slightly affected by the time delay value. The LQR without the static systems $D_{r}, D_{l}$ becomes unstable for $T=87 \mathrm{~ms}$. The response of the system with the state feedback small gain based controller shows higher overshoot and longer settling time than the proposed approach although it is also slightly affected by the time delay value. In Table I the cost function (16) for the simulation time horizon of $6 \mathrm{sec}$ is presented. The proposed approach clearly outperforms the LQR without the transformation, but even the small gain based state feedback controller for the time delays $T=0,300,450 \mathrm{~ms}$.

\section{CONCLUSIONS}

A distributed control is proposed for delay-independent stability of NCS composed of $(Q, S, R)$-dissipative systems. Key idea is to use the limited computational power at the plant side to implement a SOFIF controller. The SOFIF controller transforms the plant into a finite gain $L_{2}$ stable system. Finite gain $L_{2}$ stability is not affected by arbitrarily large constant time delay. Thus, the dissipativity property of the plant is preserved throughout the network. As a result delay-independent stability of the overall system can be guaranteed based on a dissipativity-based stability condition without time delay, between the original plant and controller. Simulations demonstrate the superior performance of the proposed approach, in a comparison between the systems with and without the SOFIF controller and a small gain based state feedback controller. Extensions to time-varying delay and packet loss are straightforward combined with existing approaches. Future research addresses the investigation of more general transformations.

\section{REFERENCES}

[1] R. J. Anderson and M. W. Spong. Bilateral control of teleoperators with time delay. IEEE Transactions on Automatic Control, 34(5):494501, May 1989.

[2] J. Baillieul and P.J. Antsaklis. Control and communication challenges in networked real-time systems. Proceedings of the IEEE, 95(1):9-28, January 2007.

[3] B. Berestesky, N. Chopra, and M. W. Spong. Discrete Time Passivity in Bilateral Teleoperation over the Internet. In Proceedings of the IEEE International Conference on Robotics and Automation ICRA'04, pages 4557-4564, New Orleans, US, 2004.

[4] S. Boyd, L. E. Ghaoui, E. Feron, and V. Balakrishnan. Linear Matrix Inequalities in System and Control Theory. Society of Industrial and applied mathematics,SIAM, 1994.

[5] G. Goodwin, D. Quevedo, and E. I. Silva. Filter Banks in Networked Control. In Proceedings of the 17th International Symposium in Mathematical Theory of Networks and Systems MTNS2006, pages -, Kyoto, Japan, 2006.

[6] K. Gu, V. Kharitonov, and J. Chen. Stability of Time-Delay Systems. Birkhäuser, 2003.

[7] D. Hill and P. Moylan. The Stability of Nonlinear Dissipative Systems. 21(5):708-711, 1976.

[8] H. K. Khalil. Nonlinear Systems. Pearson Education International, 1996.

[9] M. Kočara and M Stingl. PENNON: a code for convex nonlinear and semidefinite programming. Optimization Methods and Software, 18, 2003.

[10] F. Leibfritz. Compleib: Constraint matrix-optimization problem library - a collection of test examples for nonlinear semidefinite programs, control system design and related problems. Technical Report, Department of Mathematics, University of Trier, 2004.

[11] G. P. Liu, J. X. Mu, and D. Rees. Networked predictive control of systems with random network transmission delay - a polynomial approach. In Proceedings of the 16th IFAC World Congress, July 2005.

[12] J. Löfberg. YALMIP : A toolbox for modeling and optimization in MATLAB. In Proceedings of the CACSD Conference, http://control.ee.ethz.ch/ joloef/yalmip.php, 2004.

[13] R. Lozano, N. Chopra, and M. Spong. Passivation of Force Reflecting Bilateral Teleoperators with Time Varying Delay. In Proceedings of the 8. Mechatronics Forum, pages 954-962, Enschede, Netherlands, 2002.

[14] T. Matiakis, S. Hirche, and M. Buss. Control of networked systems using the scattering transformation, accepted for future publication. IEEE Transactions on Control Systems Technology.

[15] T. Matiakis, S. Hirche, and M. Buss. Local and remote control measures for networked control systems. In Proceedings of the 47th IEEE International Conference on Decision and Control, CDC 'O8.

[16] T. Matiakis, S. Hirche, and M. Buss. A novel input-output transformation method to stabilize networked control systems independent of delay. In Proceedings of 17th International Symposium Mathematical Theory of Networks and Systems, pages 2891-2897, 2006.

[17] G. Niemeyer and J. Slotine. Stable adaptive teleoperation. International Journal of Oceanic Engineering, 16(1):152-162, 1991.

[18] C. Scherer and S. Weiland. Linear Matrix Inequalities in Control. Eindhoven University of Technology, http://w3.ele.tue.nl/nl/mbs/cs/education/courses/disclmi, 2007.

[19] R.H. Tutuncu, K.C. Toh, and Todd M.J. Solving semidefinitequadratic-linear programs using SDPT3. Mathematical Programming Series B, 95:189-217, 2003.

[20] J. C. Willems. Dissipative Dynamical Systems - Part II: Linear Systems with Quadratic Supply Rates. Arch. Rational Mechanics Analysis, 45:352-393, 1972. 